\title{
Controlled Cavitation in Microfluidic Systems
}

\author{
Ed Zwaan, ${ }^{1}$ Séverine Le Gac, ${ }^{2}$ Kinko Tsuji, ${ }^{3}$ and Claus-Dieter Ohl ${ }^{1, *}$ \\ ${ }^{1}$ Faculty of Science and Technology, Physics of Fluids, University of Twente, Postbus 217, 7500 AE Enschede, The Netherlands \\ ${ }^{2}$ BIOS the Lab-on-a-Chip Group, MESA+ Institute for Nanotechnology, University of Twente, \\ Postbus 217, 7500 AE Enschede, The Netherlands \\ ${ }^{3}$ Shimadzu Europa GmbH, Albert-Hahn-Strasse 6-10, D-47269 Duisburg, Germany
}

(Received 20 February 2007; published 19 June 2007)

\begin{abstract}
We report on cavitation in confined microscopic environments which are commonly called microfluidic or lab-on-a-chip systems. The cavitation bubble is created by focusing a pulsed laser into these structures filled with a light-absorbing liquid. At the center of a $20 \mu \mathrm{m}$ thick and $1 \mathrm{~mm}$ wide channel, pancakeshaped bubbles expand and collapse radially. The bubble dynamics compares with a two-dimensional Rayleigh model and a planar flow field during the bubble collapse is measured. When the bubble is created close to a wall a liquid jet is focused towards the wall, resembling the jetting phenomenon in axisymmetry. The jet flow creates two counter-rotating vortices which stir the liquid at high velocities. For more complex geometries, e.g., triangle- and square-shaped structures, the number of liquid jets recorded correlates with the number of boundaries close to the bubble.
\end{abstract}

DOI: 10.1103/PhysRevLett.98.254501

Introduction. - Approximately 100 years ago it had been revealed that cavitation bubbles are a threat to almost any material in contact with high-speed flows; cavitation causes wear on pump blades, at bends in flow lines, and cavitation can disintegrate ship propellers [1-3]. One reason why cavitation is so aggressive lies in its ability to focus fluid flows to very small scales; the bubbles concentrate the energy from the fluid during their shrinkage. The violence of the bubble collapse is evident from the emission of shock waves and light [4] and their catalytic effect on chemical reactions [5]. Only recently the attention from largely free cavitation bubbles [6] has shifted towards confined bubbles [7]. Still, rather few modeling efforts are reported on confined bubble dynamics under the consideration of high Reynolds numbers, e.g., [8]. This Letter reports on an experimental study of cavitation dynamics occurring in a narrow gap and close to additional boundaries with unprecedented detail. The confining geometry is adapted from microfluidic systems [9] or so-called lab-on-a-chip devices. In these geometries low Reynolds number flows are typically found. However, due the rapidness of cavitation bubble-induced flows challenging actuation in microfluidics can be met. Here we will demonstrate that cavitation bubbles are able to actuate flows on a microsecond time scale.

Experimental setup. - These experiments are stimulated by the work of Chen et al. [10]. The group studied the interaction of a stable gas bubble with a laser-induced cavitation bubble in a $10 \mu \mathrm{m}$ thick gap of fluid. Additionally to the stroboscopic recording used in their work we adopt a framing camera operating at $1 \times 10^{6}$ frames/s (HPV-1, Shimadzu Corp., Kyoto, Japan). Also, the fluid gap is replaced by microfluidic structures, see below. The bubble is generated with an expanded beam from a pulsed and frequency-doubled Nd:YAG laser (Solo PIV, New Wave, Fremont, CA, USA). The energy of the laser pulses is in the
PACS numbers: 47.55.dp, 47.55.dd, 47.61.-k, 47.61.Jd

range $5-50 \mu \mathrm{J}$ and they have a duration of $6 \mathrm{~ns}$. The sketch, Fig. 1, shows that the laser beam is not collimated but slightly divergent; thus, after proper alignment the focus of the laser beam and the image plane of the $40 \times$ objective of the inverted microscope (CF 40, Carl Zeiss $\mathrm{GmbH}$, Göttingen, Germany) superimpose allowing infocus images of the bubble outline. A filter block reflects only the green laser light and lets pass all other wavelengths for proper illumination with a fiber lamp (ILP-1, Olympus, Zoeterwoude, The Netherlands). The reproducibility of the experiments enables to take stroboscopic pictures to record especially the bubble expansion at higher temporal resolution. Therefore, a single-frame camera (PCO Imager Intense, Kehlheim, Germany) is combined with a short flash light (11 ns flash duration, Nanolite KLM, HSPS, Germany) and a variable delay generator.

Lab-on-a chip devices are fabricated in an elastomeric material, PDMS (polydimethylsiloxane) using conven-

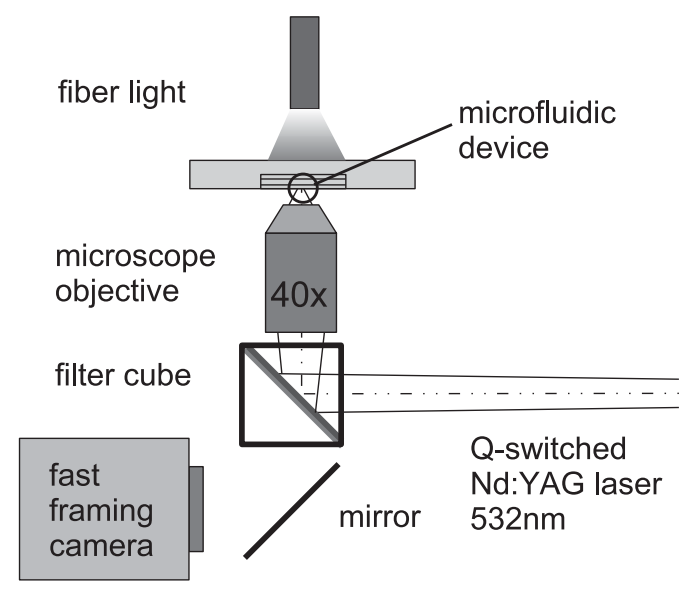

FIG. 1. Sketch of the experimental setup to generate cavitation bubbles in microfluidic devices. 
tional molding procedure [11]. Then, the chips are bonded to a microscope glass slide or a polypropylene foil after plasma activation of the surface. Chips are filled with an aqueous solution of red dye (Direct Red 81, Sigma Aldrich, St. Louis, MO, USA). For flow visualization and quantitative analysis the liquid is seeded with small particles (2 $\mu \mathrm{m}$ diameter, Duke Scientific, Fremont, CA, USA).

Bubble dynamics in radial symmetry.-The bubble dynamics in a $20 \mu \mathrm{m}$ high and $1 \mathrm{~mm}$ wide channel is undisturbed due to the large distance between the bubble and the closest channel wall; the flow field remains radial during the collapse. We observe no after bounces following the first bubble collapse and the flow stops subsequently nearly instantaneously (within $2 \mu \mathrm{s}$ ). Interestingly, the expansion of the bubble proceeds faster than its shrinkage, see Fig. 2(a) [10]. Presumably, the vapor pressure from the initial hot bubble drives the bubble expansion, but cools down as the bubble expands leading to the asymmetry in the radius-time curve. Analyzing the particle motion reveals the formation and propagation of a stagnation region during the expansion of the bubble which has been found in simulations with elastic tubes, too [12]. This region travels with a speed of approximately $20 \mathrm{~m} / \mathrm{s}$ radially outwards. From now on we discuss the collapse phase of the bubble: accounting for the fluid inertia only the bubble dynamics can be compared with the pressure driven closure of a cylindrical void. A first justification for this approach is the high Reynolds number $R e=2 R_{\max }^{2} / \nu / t_{c}$ of $R e=530$ for a bubble collapsing from $R_{\max }=43 \mu \mathrm{m}$ within $t_{c}=$ $7 \mu \mathrm{s}$; with $\nu=10^{-6} \mathrm{~m}^{2} / \mathrm{s}$ being the kinematic viscosity.
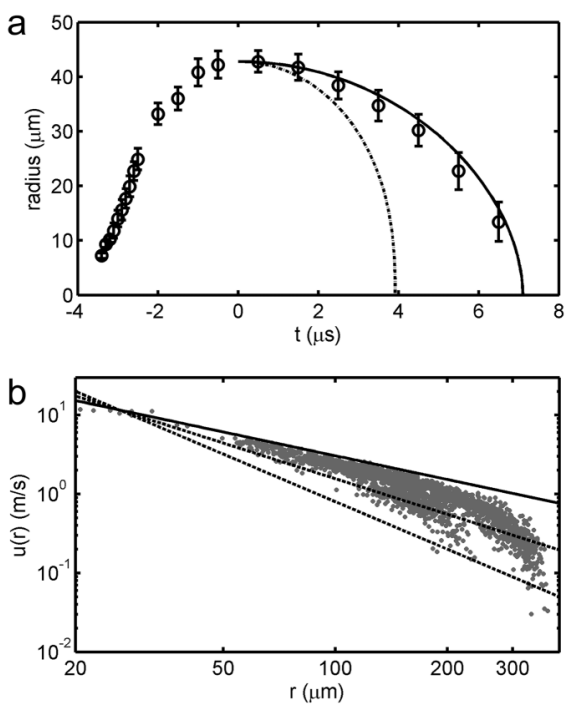

FIG. 2. (a) Comparison of the stroboscopically measured bubble radii $(O)$ and the model predictions for the 2D Rayleigh collapse (solid line) and 3D type (dashed line). The time $t=0$ is shifted to maximum bubble expansion $R_{\max }=43 \mu \mathrm{m}$. (b) The velocity is plotted in a log-log graph as a function of the distance $r$ from the bubble center. The solid line shows the predicted $1 / r$ scaling for a two-dimensional flow; the two lower dashed lines depict a $r^{-1.5}$ and a $r^{-2}$ dependency, respectively.
The planar (axisymmetric) closure of a cavity is discussed in Ref. [13] and reads:

$$
\left(R \ddot{R}+\dot{R}^{2}\right) \log \frac{R}{R_{\infty}}+\frac{1}{2} \dot{R}^{2}=\frac{p}{\rho},
$$

where $\rho$ is the liquid density, $R_{\infty}$ is the distance at which the velocity in the fluid has dropped to zero, and $p$ is the pressure far from the bubble which we assume constant, $p=10^{5} \mathrm{~Pa}$. The parameter $R_{\infty}$ comes from the fact that the velocity decreases only with $1 / r$ in the liquid; thus for a finite energy of the fluid a cutoff has to be introduced. In the current experiments this can be easily accomplished because the distance $R_{\infty}$ is identified with the geometrical constraints: the bubble is generated in the center of a $1 \mathrm{~mm}$ wide channel; thus $R_{\infty}$ is fixed to $R_{\infty}=0.5 \mathrm{~mm}$. The comparison of the measured (circles) and calculated (solid line) bubble dynamics in Fig. 2(a) shows good agreement; in contrast, the dashed line depicts the collapse dynamics for a three-dimensional bubble predicting a much faster shrinkage than observed.

It might be argued that due to the small channel height the boundary layer affects the bubble dynamics and viscosity has to be taken into account. A rough estimate of the distance, $\delta$, vorticity can grow from the wall into the channel is given by $\delta=\sqrt{\nu t}$. Thus, with a bubble lifetime of $t=11 \mu \mathrm{s}$ we estimate $\delta \approx 3 \mu \mathrm{m}$ being smaller than half the channel height of $10 \mu \mathrm{m}$.

To detail further if the bubble dynamics is indeed - as suggested by the good agreement with the 2D Rayleigh Eq. (1) - dominated by an inertial planar flow, the velocity as a function from the bubble center is analyzed.

In 2D the velocity of the liquid $u(r, t)$ should follow the assumed $1 / r$ dependency. To check this dependency, we make use of the seed particles in the flow as tracers for the local flow velocity by using a standard particle image velocimetry technique (PIV) [14] to cross correlate interrogation areas from two successive frames. With this technique the locally averaged flow velocity is measured. It is important to note that due to the large focal depth of the imaging optics not only particles in the center of the channel but also close to the bottom and top, thus within the boundary layer, are imaged. Therefore, the flow velocity obtained from the PIV algorithm is a lower bound of the flow which we expect to be highest in the center of the channel. Figure 2(b) depicts the measured velocity (gray points) in the channel just one frame prior to bubble collapse; the bubble surface is located at $r=20 \mu \mathrm{m} \pm$ $3 \mu \mathrm{m}$. The solid line denotes a curve with a $1 / r$ dependency, with $u(r)=3.06 \times 10^{-4} / \mathrm{r} \mathrm{m}^{2} / \mathrm{s}$ being a fit to the leftmost data points. This curve follows very nicely the fastest measured particles in the flow up to a distance of $r=180 \mu \mathrm{m}$. The two dashed curves depict a velocity dependency as $r^{-1.5}$ and $r^{-2}$, respectively. Although the line for $r^{-1.5}$ converges faster $(u \rightarrow 0)$ as $r \rightarrow \infty$ it underestimates the velocities closer to the bubble wall. The message from the analysis summarized in Fig. 2(b) is 
that the velocity field in the center of the channel is best represented with a planar flow field.

However, it is expected that for larger bubbles the dynamics cannot anymore be described with a simple inertia driven model, Eq. (1), possibly because viscosity has more time to diffuse from the channel walls and affects the inflow. Additionally, for larger bubbles the laser energy is increased with the chance to create more noncondensable gas which might hinder the shrinkage, too. This lengthening of the shrinkage is documented in Fig. 3 revealing that the good agreement between the 2D bubble model (solid line) gets lost for larger bubbles, here when $R_{\max }>$ $90 \mu \mathrm{m}$.

Close to a boundary. - It is well known that a bubble collapsing at some stand-off distance from an infinite boundary $[15,16]$ develops an inertia driven flow focusing phenomenon [17]. The proximity of the boundary disturbs the radial flow, e.g., it causes a faster inflow from the walldistant part of the bubble interface. There flow becomes focused and a jet flow directed towards the rigid boundary [18] is generated. This jet flow travels through the bubble center, penetrates the lower bubble interface, and impacts onto the boundary where it creates a long lasting vortex ring [19]. We now want to test if comparable dynamics is found in a planar geometry. Figure 4(a) depicts the stages of a bubble expanding and shrinking at the channel boundary, see bright lower border. During shrinkage the bubble obtains a triangular shape. The top part of the bubble interface flips towards the boundary between $t=5 \mu \mathrm{s}$ and $t=9 \mu \mathrm{s}$ and thereby creates a jet with a tip diameter of approximately $8 \mu \mathrm{m}$. It impacts with a velocity of roughly $25 \mathrm{~m} / \mathrm{s}$ onto the boundary. Having a closer look, we find very similar features which have been reported in axisymmetric collapse in planar geometry, too. One of these is the splashing phenomena [20]: liquid is accelerated away from the wall - in a sort of splash - when the radial flow from the still shrinking bubble impacts onto the outwards spreading jet flow [noticeable by the kinks of the split bubbles in frame $12 \mu \mathrm{s}$ of Fig. 4(b)]. At later times the two tiny remains of the bubbles become entrained in

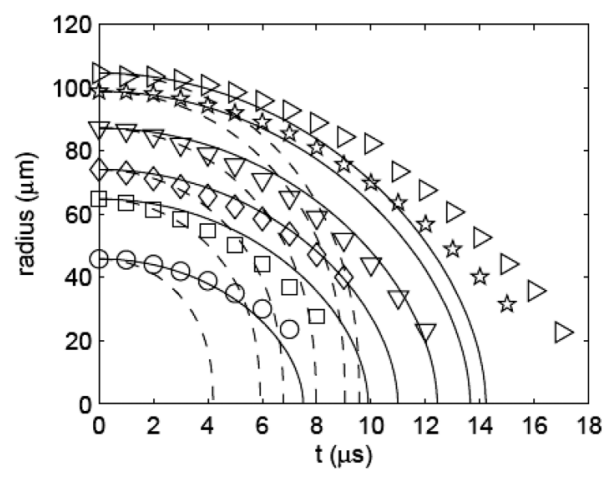

FIG. 3. Comparison of the bubble dynamics for different sized bubbles with a two-dimensional model (solid line) and the 3DRayleigh collapse (dashed line). Markers are from measurements with the high-speed camera. two counter-rotating vortex rings. The vortex rings are revealed with PIV. An averaged flow field over $40 \mu \mathrm{s}$ after the jet impact is depicted in Fig. 4(c). Tracing the maximum of the vorticity $c=\underline{\nabla} \times \underline{u}(x, y)$ within this time interval we find a decrease (not shown) from $10^{5} \mathrm{~s}^{-1}$ to $3 \times 10^{4} \mathrm{~s}^{-1}$. Thus, the center of the vortex rotates with an initial rotation rate of more the 10.000 rotations/s!

Jetting in more complex geometries. - Confined bubbles in tubes have received attention in the medical field, for example, cavitation in arteries produced in laser assisted angioplasty, e.g., [21], and in diagnostic and therapeutic ultrasound applications [22]. In channels with rectangularshaped cross sections we find that largely expanding bubbles become ellipsoidal, see Fig. 5, left. The lower row shows the resulting bubble shape after jets have developed. Here, as in the next two discussed cases jets develop always during the shrinkage and at locations of the interface where most fluid is available. For the $150 \mu \mathrm{m}$ wide channel, two jets develop in opposite directions flowing along the channel. At a later stage (not shown in Fig. 5) these jets impact and reflect towards the channel walls causing the transport of vorticity to the walls. In the second and third example of Fig. 5 again jetting is found from interface areas where liquid is most easily "accessible": a bubble oscillating inside a triangular structure develops fast jets starting from the feeding capillaries placed at the corners, Fig. 5, center. A similar observation is found in a square geometry (Fig. 5, right); here four jets start from the

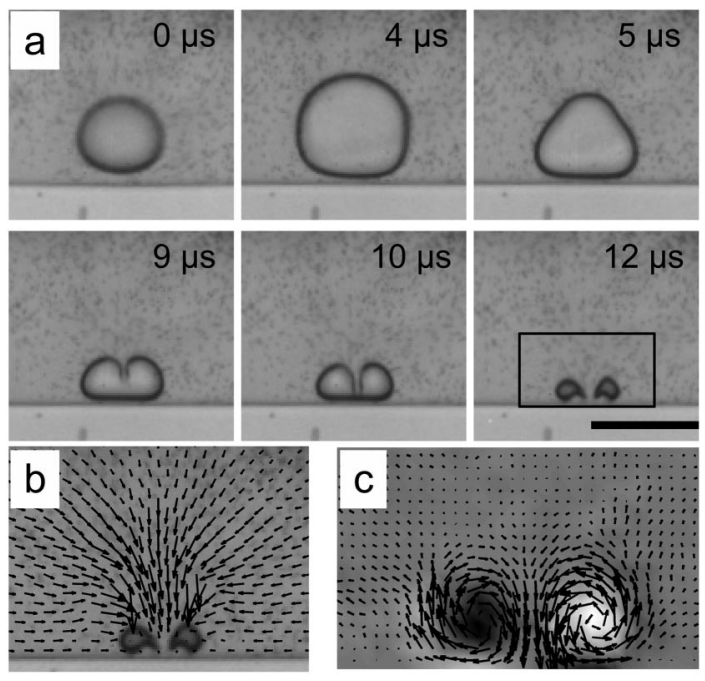

FIG. 4. Planar bubble dynamics close to a channel wall. (a) Selected frames from a high-speed recording depicting the formation of a liquid jet towards the wall. The bar indicates a length of $150 \mu \mathrm{m}$. (b) Velocity field of the flow after the bubble has split up due to the impacting jet flow. The jet flow and the radial inflow impact and lead to a splashing phenomenon (see text). The longest velocity vector corresponds to $7.4 \mathrm{~m} / \mathrm{s}$. (c) Vorticity plot after the bubble has collapsed averaged over the time interval $t \in[24 \mu \mathrm{s} ; 64 \mu \mathrm{s}]$. Note that the bubble has already vanished during this time interval. Bright colors denote counterclockwise rotation and dark ones clockwise. 

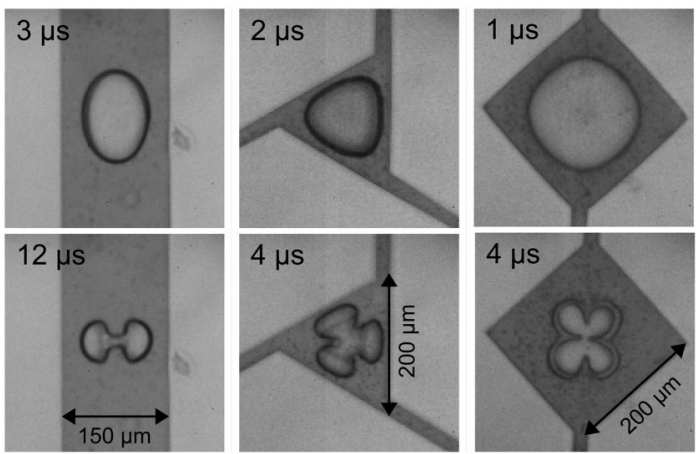

FIG. 5. Snapshots for three different geometries from left to right: single bubble cavitation in a $150 \mu \mathrm{m}$ wide and several millimeter long channel, in a $200 \mu \mathrm{m}$ isosceles triangular structure with feeding channel attached to the corners, and in a square chamber with $200 \mu \mathrm{m}$ long sides. From the time stamps it becomes evident that the jetting flow is much faster in the latter two cases.

corners, two of them being connected to feeding capillaries. This geometry leads to a bubble shape resembling a four-leaf clover. At later stage we find for the triangular and the square chambers reflections of the in-flowing jets and complex bubble breakup indicating that the planar flow field becomes lost.

Discussion and conclusions. - In general, the essentially planar bubble dynamics presented in this Letter resembles the one observed in spherical and axisymmetry. Far from boundaries the collapse of the bubble can be described with a radial velocity potential leading to a $1 / r$ drop of the velocity as compared to the $1 / r^{2}$ dependency in spherical symmetry. Yet, larger bubbles (in our case, above $R_{\max }>$ $90 \mu \mathrm{m}$ ) collapse slower, and this is likely due to viscous effects. Although we have emphasized that the bubbles exhibit an essentially planar flow-as the collapse progresses - the bubble will shrink to a size smaller than the channel height. Then a three-dimensional flow will set in. Cavitation in microfluidics allows for reaching a regime of high $R e$ numbers for microsecond time scales. The important finding that jetting and creation of vorticity occur has immediate practical applications. Jetting flows can be utilized for microfluidic pumping [23] and vorticity creation for vigorous mixing on short time scales. A visualization of the flow field is much easier to achieve in a planar geometry than in axisymmetry [24] which is demonstrated in the nicely resolved vortex structures generated after the jet impact, Fig. 4. Cavitation is a tool which allows for scaling down fluid handling to a microfluidic environment preserving the benefits of high- $R e$-number flows. The added benefit of laser created bubbles is the noninvasive generation mechanism. No connecting wires or actuators are necessary and the position of actuation can suitably addressed by scanning the laser spot with mirrors.

The authors are grateful for the discussions with A. Prosperetti, D. Lohse, and E. Klaseboer. We thank J. Li for Si-mold fabrication. C. D. O. is supported by N.W. O. VIDI (The Netherlands).

*Present address: Division of Physics and Applied Physics, School of Physical and Mathematical Sciences, Nanyang Technological University, Singapore 637551, Singapore. Electronic address: c.d.ohl@utwente.nl

[1] D. Silberrad, Engineering Journal 12, 33 (1912).

[2] M. S. Plesset and A. Prosperetti, Annu. Rev. Fluid Mech. 9, 145 (1977).

[3] T.G. Leighton, The Acoustic Bubble (Academic, New York, 1994); C.E. Brennen, Cavitation and Bubble Dynamics (Oxford University Press, New York, 1995).

[4] M. P. Brenner, S. Hilgenfeldt, and D. Lohse, Rev. Mod. Phys. 74, 425 (2002).

[5] K. S. Suslick et al., Phil. Trans. R. Soc. A 357, 335 (1999).

[6] D. Lohse, Phys. Today 56, No. 2, 36 (2003).

[7] V.S. Ajaev and G. M. Homsy, Annu. Rev. Fluid Mech. 38, 277 (2006).

[8] H. Yuan, H. N. Oğuz, and A. Prosperetti, Int. J. Heat Mass Transf. 42, 3643 (1999).

[9] T. M. Squires and S. R. Quake, Rev. Mod. Phys. 77, 977 (2005).

[10] Y.H. Chen, H. Y. Chu, and L. I, Phys. Rev. Lett. 96, 034505 (2006).

[11] A silicon mold is first fabricated in a clean-room environment using photolithography and dry-etching techniques (Bosch process). A mixture of a PDMS prepolymer and a curing agent (10:1) (Sylgard 184, Dow Corning) is subsequently poured on the resulting mold and cured for 2 hours at $60^{\circ} \mathrm{C}$. After curing, the PDMS slab is removed from the mold and reservoirs are punched using a needle.

[12] T. Ye and J. L. Bull, J. Biomech. Eng. 128, 554 (2006).

[13] H. N. Oğuz and A. Prosperetti, J. Fluid Mech. 257, 111 (1993); D. Lohse et al., Phys. Rev. Lett. 93, 198003 (2004).

[14] We use the package URAPIV from http://urapiv.wordpress. $\mathrm{com} /$. Two consecutive images are evaluated on $16 \times 16$ pixels interrogation windows with 4 pixels overlap.

[15] M.S. Plesset and R. B. Chapman, J. Fluid Mech. 47, 283 (1971).

[16] W.Lauterborn and H. Bolle, J. Fluid Mech. 72, 391 (1975).

[17] M. P. Brenner, Nature (London) 403, 377 (2000).

[18] A. Philipp and W. Lauterborn, J. Fluid Mech. 361, 75 (1998).

[19] T. B. Benjamin and A. T. Ellis, Phil. Trans. R. Soc. A 260, 221 (1966).

[20] E. A. Brujan et al., Phys. Fluids 14, 85 (2002).

[21] A. Vogel et al., Appl. Phys. B 62, 173 (1996).

[22] P. Zhong, Y. Zhou, and S. Zhu, Ultrasound Med. Biol. 27, 119 (2001); T. Ye and J. L. Bull, J. Biomech. Eng. 126, 745 (2004); E. Sassaroli and K. Hynynen, Appl. Phys. Lett. 89, 123901 (2006).

[23] B. C. Khoo, E. Klaseboer, and K. C. Hung, Sens. Actuators A, Phys. 118, 152 (2005); K. S. F. Lew, E. Klaseboer, and B. C. Khoo, Sens. Actuators A, Phys. 133, 161 (2007).

[24] A. Vogel, W. Lauterborn, and R. Timm, J. Fluid Mech. 206, 299 (1989). 